\title{
CARACTERIZACIÓN EN NIÑOS DE 6 AÑOS DE EDAD CON INTERFERENCIAS DENTARIAS DE ESCUELAS PRIMARIAS
}

\section{CHARACTERIZATION IN CHILDREN 6 YEARS OF AGE WITH TOOTH INTERFERENCE OF PRIMARY SCHOOLS}

\author{
Hechavarría- Martínez Bárbara ${ }^{1 a}$, Núñez-Antúnez Leonardo ${ }^{1 b}$, Pons-Hechavarría \\ Leanne $^{1 \mathrm{~b}}$, Núñez-Almarales Nayra. ${ }^{1 \mathrm{~b}}$
}

\section{RESUMEN}

Objetivo: Caracterizar a los niños de 6 años de edad de escuelas primarias, pertenecientes al área de salud del Policlínico Docente "30 de Noviembre". Materiales y métodos. Se realizó un estudio observacional descriptivo y de corte transversal con el objetivo de. El universo estuvo constituido por 83 niños cuya atención estomatológica se realiza en el departamento de estomatología del Policlínico Docente "30 de Noviembre", en el período comprendido entre Junio de 2017 y Abril de 2018 . Para la obtención de los datos se realizó una entrevista estructurada a padres o tutores, con el fin de obtener datos precisos y verificados, utilizando como medida de resumen el porcentaje y reflejando los datos en tablas de contingencia. Se realizó el examen bucal a los niños utilizando el depresor lingual y la luz natural o artificial. Resultados: En el estudio predominó el sexo femenino. Los hábitos deformantes más practicados fueron los hábitos posturales y la succión digital. Conclusiones: La anomalía más encontrada fue la oclusión invertida posterior unilateral. Teniendo en cuenta esto se recomienda ampliar la investigación en otros centros escolares, ya que estos resultados nos alertan sobre la necesidad de interceptar las interferencias dentarias desde edades tempranas.

Palabras Clave: Niños; Hábitos; Mal oclusiones. (Fuente: DeCS BIREME)

\section{ABSTRACT}

Objective: Characterize children from 6 years of age in primary schools belonging to the health area of the Policlinico Docente "30 November". Methods: He was an observational study descriptive and cross-sectional aiming to. The universe was composed of 83 children whose dental care is carried out in the Department of Stomatology of the Policlinico teacher "30 de Noviembre", in the period between June of 2017 and 2018 April. The data was an interview structured to parents or guardians, in order to obtain data accurate and verified, using as a summary measure the percentage and reflecting the data in contingency tables. He was the oral test to children using the tongue depressor and natural or artificial light. Results: The study dominated the female sex. The most practiced disfiguring habits were postural habits and digital suction. Conclusions: Most found anomaly was one-sided after reversed occlusion. Taking into account this recommended expanding research in other schools, since these results alert us to the need to intercept the tooth interference from an early age.

Keywords: Children; habits; malocclusion. (Source: MeSH NLM)

Recibido: 24 de julio de 2018

Aprobado: 26 de setiembre de 2018

Publicado: 30 de setiembre de 2018

'Facultad de Estomatología, Santiago de Cuba, Cuba.

${ }^{a}$ Especialista de 2do grado en Estomatología General Integral.

${ }^{\mathrm{b}}$ MSc. Salud Bucal Comunitaria.

Este es un artículo Open Access distribuido bajo la licencia Creative Commons Atribución-NoComercial- Compartir Igual 4.0

Correspondencia:

Bárbara Olaydis Hechavarría Martínez

Correo electrónico: barbaraolaidis@infomed.sld.cu

Citar como: Hechavarría- Martínez B, Núñez-Antúnez L, Pons-Hechavarría L, Cúñez-Almarales N. Caracterización en niños de 6 años de edad con interferencias dentarias de escuelas primarias. KIRU. 2018; 15(3): 115 - 120. https://doi.org/10.24265/kiru.2018.v15n3.02 


\section{INTRODUCCIÓN}

La cara en crecimiento es una estructura maravillosamente compleja, cuyo desarrollo, mucho más que un mero incremento de tamaño deviene un proceso delicadamente equilibrado, que gradualmente modela y da nueva forma al rostro del niño hasta convertirse en el del adulto ${ }^{(1)}$.

Cuando el niño nace, los estímulos ambientales que producen respuesta a nivel de la cara son: la respiración nasal, la lactancia materna, la abrasión, desgaste de la primera dentición, así como, la erupción de los incisivos y primeros molares permanentes ${ }^{(2)}$.

El tiempo de duración de la dentición primaria es corto, de unos 5 años, su desarrollo y función lo imprime el tipo de dieta, generando gran actividad de los maxilares y estos 20 dientes sufren cambios tales como la atrición y los diastemas ${ }^{(3)}$.

En cuanto a la dentición mixta, esta se establece en el arco dentario con el brote de los primeros molares permanentes; ya que detrás de estos, continúa el crecimiento de los maxilares para alojar a los molares complementarios; las apófisis alveolares aumentan su altura conjuntamente con el resto de la cara, este crecimiento está íntimamente relacionado con las adaptaciones continuas de las relaciones oclusales ${ }^{(4)}$.

Tanto la dentición temporal como la mixta, pueden presentar alteraciones, tales como, disminución del ancho de las arcadas dentarias, mal posiciones dentarias y algunos hábitos deformantes que pueden provocar desviaciones de la mandíbula que, de no ser interceptadas, interferirá el crecimiento y el desarrollo de la mandíbula $y$ de las articulaciones temporomandibulares (ATM), que afectan la función y estética del paciente ${ }^{(5)}$.

Las interferencias oclusales, que constituye un problema habitual en las denticiones temporal y mixta, se asocian a maloclusiones funcionales como mordidas cruzadas anteriores y posteriores, anomalías faciales, mordidas abiertas anteriores, desviaciones de la línea media y afectaciones de la articulación temporomandibular, ya que, obstaculizan las relaciones que se establecen entre los arcos dentarios impidiendo el trayecto armonioso de la mandíbula desde la posición postural hasta los movimientos de la dinámica mandibular, los que no son tratados a tiempo pueden provocar alteraciones esqueletales ${ }^{(6)}$.

La ortodoncia moderna incluye la intercepción de las anomalías oclusales, como un aspecto muy importante, y dentro de éstas, que sean detectadas inmediatamente el tratamiento de las interferencias oclusales ${ }^{(7,8)}$.

En las encuestas epidemiológicas del Programa de Atención al menor de 19 años del área de salud de la Clínica Estomatológica Provincial Docente de Santiago de Cuba, se estimó que en 705 niños de 6 años de edad el $52 \%$ presentaban interferencias dentarias y el 10,4\% mostraron anomalías de oclusiones invertidas por lo que es un hecho que ellas pueden constituir un riesgo en el establecimiento de anomalías de la oclusión en los niños portadores de las mismas ${ }^{(9,10)}$.

Teniendo en cuenta que en nuestra área de salud no existen investigaciones sobre el tema, surgió la necesidad de investigar las características de los niños de 6 años de edad de las escuelas primarias con interferencias dentarias; para mejorar el estado de salud bucal de los niños mediante acciones de promoción y prevención de salud por lo que se plantea el siguiente problema científico.

\section{MATERIALES Y METODOS}

La investigación se llevó a cabo teniendo en cuenta consideraciones éticas relacionadas con los pacientes objeto de estudio, para lo cual se siguieron las normas planteadas en la declaración de Helsinki y previa autorización de la Dirección Municipal de Educación para trabajar en las escuelas primarias pertenecientes al área de salud del Policlínico Docente "30 de noviembre". Se solicitó consentimiento informado de padres y/o tutores de los pacientes objeto de estudio por ser menores de edad, previa información del contenido del trabajo.

Se realizó una revisión bibliográfica exhaustiva en la biblioteca de la Clínica Estomatológica Provincial Docente y en el Departamento de Maestría de Salud, así como las disponibles en revistas y sitios de Internet avalados científicamente, libros, tesis y otros documentos. Para la obtención de la información se realizó la guía de entrevista estructurada a padres y/o tutores con el fin de obtener datos precisos y verificados.

Para completar la ficha de vaciamiento de datos, se procedió a realizar un examen clínico intrabucal y funcional en los departamentos de enfermería de las propias escuelas y otros locales acondicionados para ello. Con la participación de 2 estomatólogos y 2 estudiantes, además de las enfermeras de dichos departamentos. 
Se realizó un estudio observacional, descriptivo y transversal en niños de 6 años de edad pertenecientes a las escuelas primarias del área de salud del Policlínico Docente "30 de Noviembre" del Municipio Santiago de Cuba: "José Mercerón", "Miguel Ángel Oramas", "Arturo Hung", "Gilfredo Rondón", "Vietnam Heroico", "Rafael Espinosa", "Rafael Millán" y seminternado "Manuel Ascunse Domenech"; cuya atención estomatológica se lleva a cabo en el departamento de estomatología de dicho policlínico. El estudio se procede a ejecutar con el objetivo de caracterizar a los niños con interferencias dentarias según variables de interés.

El universo de estudio estuvo constituido por 83 niños de 6 años de edad de ambos sexos que presentaron interferencias dentarias, pertenecientes a las escuelas primarias del área de salud del Policlínico Docente " 30 de noviembre" los mismos se encontraban en dentición temporal y mixta temprana.

Para lograr el objetivo propuesto se tuvieron en cuenta las siguientes variables:

\section{Sexo}

2. Lactancia.

3. Hábito postural.

4. Hábito uso del biberón.

5. Función Respiración

6. Tipo de dentición.

7. Diente temporal con presencia de interferencias

Se hizo una revisión bibliográfica exhaustiva en la biblioteca de la Clínica Estomatológica Provincial Docente y en el Departamento de Maestría de Salud, así como la búsqueda de revistas y sitios de Internet avalados científicamente, las disponibles, también en libros, tesis y otros documentos. Para la obtención de la información se fabricó la guía de entrevista estructurada a padres $\mathrm{y} / \mathrm{o}$ tutores con el fin de obtener datos precisos y verificados. Luego, se procedió a realizar un examen clínico intrabucal y funcional en los departamentos de enfermería de las propias escuelas y otros locales acondicionados para ello.

El método de análisis y síntesis se aplicó para el estudio detallado de la información procesada, sirvió para establecer nexos entre las variables $y$ caracterizar la entidad estudiada.

La información obtenida se procesó utilizándose el porcentaje como medida de resumen. Los resultados obtenidos se presentaron en tablas de contingencia.

\section{RESULTADOS}

En la tabla 1 se observa un número significativo de niños que presentaron interferencias dentarias, 83 para un $43.0 \%$, con predominio del sexo femenino (49 escolares para un $25.4 \%)$.

Tabla 1. Niños de 6 años de edad con interferencias dentarias según sexo.

\begin{tabular}{|c|c|c|c|c|c|c|}
\hline \multirow{3}{*}{ Sexo } & \multicolumn{4}{|c|}{ Interferencias dentarie } & \multicolumn{2}{|c|}{ Total } \\
\hline & \multicolumn{2}{|c|}{ SI } & \multicolumn{2}{|c|}{ NO } & \multirow[b]{2}{*}{ № } & \multirow[b]{2}{*}{$\%^{*}$} \\
\hline & № & $\%^{*}$ & № & $\%^{*}$ & & \\
\hline Femenino & 49 & 25.4 & 59 & 30.6 & 108 & 56.0 \\
\hline Masculino & 34 & 17.6 & 51 & 26.4 & 85 & 44.0 \\
\hline Total & 83 & 43.0 & 110 & 57.0 & 193 & 100 \\
\hline
\end{tabular}

Fuente: Encuesta

*\% calculado en base al total de niños examinados.

En la tabla 2, se muestra la relación de niños con interferencias dentarias según tipo de dentición y sexo, se encontró un predominio de dentición mixta temprana, observándose en un total de 53 niños, lo que representa el $63.9 \%$ de la muestra examinada. El mayor porcentaje en este grupo eran niñas.

Podemos observar en la tabla 3, el tipo de lactancia que recibieron los niños, predominando la lactancia no adecuada, en 53 niños para un $63.8 \%$, siendo más acentuada en el sexo femenino.

La relación de hábitos deformantes y sexo se presenta en la tabla 4, se muestra un predominio de los hábitos posturales en un total de 26 niños (31.3\%), seguido de la succión digital con 21 niños (25.3\%), principalmente en niños del sexo femenino.

En la tabla 5 se muestra la relación de niños con interferencias dentarias según anomalías y sexo, en el cual se observa que el mayor número de niños presentaron oclusión invertida posterior funcional, en un total de 37 niños, lo cual representa un $44.5 \%$ de esta muestra, seguido de la anomalía pseudo clase III funcional, presente en 21 escolares para $25.3 \%$. 
Tabla 2. Medidas de resumen del volumen de la vía aérea y de la posición antero-posterior del hueso hioides

\begin{tabular}{lllllll}
\hline \multirow{2}{*}{ Tipos de lactancia } & \multicolumn{2}{c}{ Sexo } & \multicolumn{2}{c}{ Masculino } & \multicolumn{2}{c}{ Total } \\
& № & $\%$ & № & $\%^{*}$ & № & $\%$ \\
\hline Temporal & 16 & 19.2 & 14 & 16.9 & 30 & 36.1 \\
Mixta temprana & 33 & 39.8 & 20 & 24.1 & 53 & 63.9 \\
Total & 49 & 59.0 & 34 & 41.0 & 83 & 100
\end{tabular}

\% calculado en base al total de niños con interferencias

Tabla 3. Niños con interferencias dentarias según tipo de lactancia y sexo

\begin{tabular}{lllllll}
\hline \multirow{2}{*}{ Tipos de lactancia } & \multicolumn{2}{c}{ Sexo } & \multicolumn{2}{c}{ Masculino } & \multicolumn{2}{c}{ Total } \\
& № & $\%$ & № & $\%^{*}$ & № & $\%$ \\
\hline No adecuada & 32 & 38.5 & 21 & 25.3 & 53 & 63.8 \\
Adecuada & 17 & 20.5 & 13 & 15.7 & 30 & 36.1 \\
Total & 49 & 59.0 & 34 & 41.0 & 83 & 100 \\
\hline
\end{tabular}

Tabla 4. Niños con interferencias dentarias según hábitos y sexo

\begin{tabular}{lllllll}
\hline \multirow{2}{*}{ Hábitos } & \multicolumn{2}{c}{ Femenino } & \multicolumn{2}{c}{ Masculino } & \multicolumn{2}{c}{ Total } \\
& № & $\%^{*}$ & № & $\%^{*}$ & № & $\%^{* *}$ \\
\hline Succión digital & 15 & 71.4 & 6 & 28.5 & 21 & 25.3 \\
Biberón & 6 & 46.1 & 7 & 53.8 & 13 & 15.6 \\
Disfunción respiratoria & 8 & 50.0 & 8 & 50.0 & 16 & 19.2 \\
Hábitos posturales & 19 & 73.0 & 7 & 26.9 & 26 & 31.3 \\
\hline$*$ En base al total de niños & & & & & &
\end{tabular}

**\% En base al total de niños

Tabla 5. Niños con interferencias dentarias según anomalías y sexo

\begin{tabular}{|c|c|c|c|c|c|c|}
\hline \multicolumn{7}{|c|}{ Sexo } \\
\hline \multirow[t]{2}{*}{ Anomalías } & \multicolumn{2}{|c|}{ Femenino } & \multicolumn{2}{|c|}{ Masculino } & \multicolumn{2}{|c|}{ Total } \\
\hline & № & $\%^{*}$ & № & $\%^{*}$ & № & $\%$ ** \\
\hline Mordida abierta & 9 & 64.2 & 4 & 40.0 & 13 & 15.6 \\
\hline Pseudo clase III funcional & 12 & 57.1 & 9 & 42.8 & 21 & 25.3 \\
\hline Oclusión invertida posterior unilateral & 25 & 67.5 & 12 & 32.4 & 37 & 44.5 \\
\hline Sin anomalías & 7 & 58.3 & 5 & 41.6 & 12 & 14.4 \\
\hline
\end{tabular}

*\%En base al total de anomalías por fila.

*\%En base al total de niños. 


\section{DISCUSIÓN}

Ruiseco et al. (11) en su estudio sobre las interferencias oclusales en niños de pre escolar y primer grado encontraron 84 niños con interferencias dentarias en dentición mixta temprana y 59 niños afectados con interferencias en dentición temporal, obteniendo resultados similares a nuestro estudio.

En nuestra investigación un mayor predominio de interferencias oclusales en el sexo femenino pudiera estar atribuido a que la población femenina supera a la masculina en nuestro país a esta edad, existiendo una relación de 995 personas del sexo masculino por cada 1000 de la población femenina, pues la literatura consultada no refleja relación directa entre la aparición de interferencias dentarias y el sexo.

Estos resultados difieren a los mostrados por González G, Ardanza $P .{ }^{(12)}$, quienes encontraron un mayor número de interferencias oclusales en niños con dentición temporal (34, para 42,5\%) sin considerar el sexo.

Los resultados de esta tabla pudieran atribuirse que nuestro estudio abarca niños de 6 años de edad y es donde comienza el brote del primer molar permanente dando lugar al inicio de la dentición mixta temprana y una mayor relación con la aparición de interferencias en estos escolares que en dentición temporal.

La masticación es de suma importancia para mantener el equilibrio dinámico de la boca es la principal puerta de entrada energética y uno de los elementos más importantes en el desarrollo de la cara.

La persistencia de dieta blanda altera el desarrollo del sistema estomatognático y sus funciones como, habla, respiración, proceso de ingestión de alimentos, entre otras.

La leche materna se debe suministrar como el único alimento hasta los 6 meses de vida del niño/a, ya que contribuye de forma eficaz a su desarrollo físico, intelectual y social. A. I. Kapandji ${ }^{(13,14) \text {. }}$

Debemos aclarar que estos resultados nos alertan sobre la necesidad de incrementar las acciones de promoción y prevención de salud en conjunto con el médico de la familia, la enfermera y los factores sociales para concientizar a las madres sobre la importancia de la lactancia materna.

González Valdés ${ }^{(15)}$ en su estudio, encontró cifras similares donde hubo predominio de la lactancia no adecuada en el $62.5 \%$ de los niños con interferencias dentarias.
La lactancia materna estimula el desarrollo y maduración de las funciones del aparato bucal y contribuye en la prevención de anomalías dento-buco-maxilo-faciales. La Organización mundial de la salud recomienda que la alimentación materna debe ser exclusiva los primeros 6 meses de vida y luego debe ser complementada con otros alimentos hasta los 2 años.

Los hábitos son patrones de contracción muscular de naturaleza compleja que se aprenden y que al repetirse llegan a convertirse en inconscientes, pueden ser beneficiosos cuando sirven como estímulo para el crecimiento normal de los maxilares, ejemplo de ello es la acción normal de los labios y la adecuada masticación, o perjudiciales cuando interfieren con el patrón regular de crecimiento facial y pueden llegar a producir anomalías dento-maxilofaciales.

Comparado con la investigación de Galarza E. ${ }^{(16)}$, los hábitos posturales fueron encontrados en un $26,2 \%$ de los educandos con interferencias dentarias, cifra que difiere de las encontradas en el presente estudio, mientras que el hábito de succión digital estuvo presente solo en el $10.0 \%$ de quienes presentaron interferencia.

Los resultados en la investigación pueden obedecer a que las malas posturas que adoptan los niños en la infancia durante el horario del sueño, de lectura y actividades diurnas pueden traer como resultado la aparición de alteraciones oclusales y deformidades faciales, en el caso de la succión digital existen algunos factores que la condicionan como: la falta de cariño, hambre, ansiedad o inducidos por los padres.

Los resultados de la presente investigación coinciden con los de Zúñiga y Caza ${ }^{(17)}$ en cuyo estudio prevaleció la mordida cruzada posterior unilateral con un $63.6 \%$ sin distinción de sexo. Otro estudio obtuvo que de 144 niños examinados 39 presentaron interferencias oclusales, de ellos el $66.67 \%$ tenían mordida cruzada posterior, dato similar a los obtenidos en el presente estudio ${ }^{(18)}$

La maloclusión dental es la alineación incorrecta de los dientes. Puede aparecer por anomalías de tamaño o de posición de los dientes, del tamaño relativo de las arcadas dentarias y su alineación, o de los tipos de relaciones oclusales. ${ }^{(19,20)}$ Este resultado puede estar relacionado con los hábitos posturales y la presencia de interferencias dentarias.

Se puede concluir que, en los niños de escuelas primarias de 6 años de edad, con interferencias dentarias del "Policlínico Universitario Julián Grimau García", predominó 
el sexo femenino y el periodo de dentición mixta temprana. Los hábitos posturales y la succión digital fueron los hábitos deformantes más practicados. La anomalía predominante en los niños estudiados fue la oclusión invertida posterior unilateral, pudiendo estar influenciada por la dieta y la lactancia no adecuadas.

Contribuciones de autoría: EMB, NAL, PHL, NAN participaron en el diseño del estudio, recolección de los datos, análisis e interpretación de resultados. Redacción y revisión crítica del artículo. Aprobación de la versión final del artículo.

Fuente de financiamiento: El estudio fue financiado por los autores.

Conflicto de intereses: Los autores no han declarado conflicto de intereses en este artículo.

\section{REFERENCIAS}

1. Wheeler. Anatomía, fisiología y oclusión dental - 10th Edition [libro electrónico], Las Vegas, NV Elsevier 22 jun. 2015 [Consultado: Jun 2018]. Disponible en: https://www.elsevier.com/books/wheeler-anatomiafisiologia-y oclusiondental/nelson/978-84-9022-920-0.

2. Otaño Lugo R. Manual clínico de Ortodoncia. La Habana: Editorial Ciencias Médicas; 2013.

3. Arias M, Soto L. Desgastes selectivos en el tratamiento de oclusiones cruzadas unilaterales funcionales. Rev. Haban. Cienc. Méd. [Internet] 2004 [Citado: 16 de enero de 2017]; 3(8):0-5. Disponible en: http://www.redalyc.org/articulo.oa?id=180426080002

4. Ferreiro A. Tratamiento de interferencias oclusales en niños de 3 a 7 años. Policlínico 19 de abril. Rev. Cubana Estomatol.2015 [citado 12 de diciembre de 2017]. Disponibleen:http://estomatologia2015.sld.cu/index.php/e stomatologia/nov2015/ paper/view File/891/390.

5. Cabrera Sánchez TV, Martínez Ramos MR, Comas Mirabent R, González Esplanger L, Perú Seguí Y. Interferencias oclusales en niños con dentición temporal y mixta temprana. MEDISAN. 2015 [citado: 25 de febrero de 2017]; 19(3).

Disponible en: http://scielo.sld.cu/scielo.php?script=sci_arttext\&pid=S 1029-30192015000300005\&lng=es

6. Cabrera T, Sánchez I, George Y, Martínez M, Ramírez Y, González L, Estado de la oclusión y tratamiento selectivo en niños con dentición temporal y mixta temprana, MEDISAN 2016;20(3):289

7. Torres M. Persistencia de dieta blanda en niños que asisten a un servicio de odontología pediátrica de Cali. Revista Areté. 2015;15(2):53-8.

8. Ministerio de Salud (MINSAL), Gobierno de Chile. Lactancia Materna [Citado: 26 de diciembre de 2011] Disponible en:http://www.minsal.gob.cl/portal/url/page/minsalcl/g_pro teccion/g_lactanciamaterna/saludinmigrantespresentacion .html
9. Vergara R, Barrueco L, Díaz del Mazo L, Pérez E, Sánchez T. Influencia de la lactancia materna sobre la aparición de maloclusiones en escolares de 5 a 6 años. MEDISAN [Internet]. 2014 Ago [citado 16 julio 2017 ] ; 18( 8 ): 1064-1070. Disponible en: http://scielo.sld.cu/scielo.php?script=sci arttext\&pid=S 1029-30192014000800005\&lng=es.

10. Pino V José Luis, López E Miguel Ángel, Medel I Andrea Pilar, Ortega S Alejandra. Factores que inciden en la duración de la lactancia materna exclusiva en una comunidad rural de Chile. Rev. chil. nutr. [Internet]. 2013 Mar [citado 2017 julio 10] ; 40( 1 ): 48-54. Disponible

en: https://scielo.conicyt.cl/scielo.php?script=sci_arttext\&pi d=S0717-

$75182013000100008 \&$ Ing=es. http://dx.doi.org/10.406 7/S0717-75182013000100008.

11. Ruiseco A, Llanes M, Lázaro O, Rodríguez A. El dolor articular y su relación con las interferencias oclusales. Revista Habanera de Ciencias Médicas 2014;13(3):417-424.

12. González G, Ardanza P. Rehabilitación Protésica Estomatológica. Ciudad de La Habana: Editorial Ciencias Médicas; 2013.

13. Kapandji, I., "Fisiología articular: esquemas comentados de mecánica humana. Tomo II: Miembro Inferior". Editorial Médica Panamericana, 2002.

14. Real Academia Española (RAE) (2014). [En línea]. [Consultado 12 de junio de 2017]. Disponible en: http://dle.rae.es/

15. González D, Alemán PC, Delgado Y, Díaz L, L E. Presencia de mordida invertida en un grupo de niños y adolescentes. San Antonio de los Baños. 2014. Rev haban cienc méd [Internet]. 2015 Jun [Citado: 11 de julio de 2017]; 14(3): 270-280.. Disponible en: http://scielo.sld.cu/scielo.php?script=sci_arttext\&pid=S 1729- 519X2015000300004\&lng=es

16. La salud en el colegio. www.larioja.com/larioja/201409/08/salud-colegio-20140908135513.html

17. Zuñiga $\mathrm{M}, \mathrm{Caza} \mathrm{H}$. Malos Hábitos y herencia genética como factores etiológicos de la maloclusión en niños de 5 a 13 años que fueron atendidos en el hospital brigada 11 "Galápagos" de la ciudad de Riobamba en el período de junio-diciembre 2013. [tesis de grado]. Riobamba: Universidad Nacional de Chimborazo, Carrera de odontología; 2014.

18. Pérez I, Pedraza M, Robles L. Manejo estomatológico en paciente con trastorno espectro autismo. Reporte de caso.https://www.actaodontologica.com/ediciones/201 6/1/art-11/

19. Valarezo T, Castillo A. Hábitos bucales deformantes en niños de tres escuelas del barrio Motupe de la ciudad de Loja. Período marzo- julio 2016. [Tesis para optar por el título profesional de odontólogo]. Loja: Carrera de odontología, Universidad Nacional de Loja; 2016.

20. Sandoval P, Bizcar B. Beneficios de la Implementación de Ortodoncia Interceptiva en la Clínica Infantil. Int. J. Odontostomat. [Internet]. 2013 Ago [citado: 23 de junio de 2018 ] ; 7( 2 ): 253-265. Disponible en: https://scielo.conicyt.cl/scielo.php?script=sci_arttext\&pi $\mathrm{d}=S 0718-381 \times 2013000200016 \&$ Ing $=$ es. http://dx.doi.org/10.4067/S0718-381X2013000200016. 